\title{
Perspectivas éticas en el manejo de la pandemia COVID-19 y de su impacto en la salud mental
}

\author{
Ethical perspectives on the management of COVID-19 pandemic and its impact on mental health
}

\author{
Renato D. Alarcón-Guzmán ${ }^{1,2}$
}

\section{RESUMEN}

En tanto que componente fundamental del quehacer médico a lo largo de la historia, la Ética es aún más prominente en situaciones de emergencia en salud pública. Luego de exponer los principios éticos esenciales y de enfatizar su valor en el cuidado médico de todo ser humano, se plantea la inevitable tensión que su aplicación genera en los diversos contextos de la relación médico-paciente. Se presta especial atención a las distinciones de la perspectiva ética en casos individuales y en el ámbito colectivo o de salud pública, particularmente en el curso de emergencias masivas como la desencadenada por COVID-19. A la luz de recientes documentos nacionales e internacionales, se discute el inevitable advenimiento de nuevas normas que deberán incluir aspectos socio-culturales, psicobiológicos y legales poseedores de un impacto clínico altamente significativo.

Palabras clave: Ética médica, emergencias sanitarias, pandemia, salud pública, salud mental.

\section{SUMMARY}

As an essential component of medical work throughout history, Ethics becomes even more prominent in public health emergency situations. After presenting the fundamental ethical principles, and emphasizing their value in the medical care of every human being, the inevitable tensions that their application generates in the diverse contexts of the doctor-patient relationship, are examined. Particular attention must be paid to the distinctions between ethical perspectives of individual cases and those related to public health situations such as the massive emergency triggered by the COVID-1 pandemic. On the basis of recent national and international documents, the unavoidable upcoming of new ethical norms that must include socio-cultural, psychological and legal aspects of a highly significant clinical impact, is discussed.

Keywords: Medical Ethics, health emergencies, pandemic, public health, mental health.

\section{INTRODUCCIÓN}

La disciplina que conocemos como Ética se ha vinculado a los principios y a la práctica de la Medicina desde los inicios históricos de nuestra profesión. La definición de Ética Médica como núcleo epistemológico de principios morales aplicados a la práctica clínica y heurística de la medicina cubre una amplia gama de conceptos-guía, y acciones específicas con respeto, honestidad, cuidado, integridad, protección y confidencialidad como componentes básicos de una serie de pronunciamientos y documentos emitidos por organizaciones internacionales (1). Por otro lado, es conocido el hecho de que la vigencia de sus postulados se multiplica en términos de visibilidad, significado y valor cuando las circunstancias del ejercicio médico llegan a extremos de gravedad y urgencia. La pandemia de COVID-19 es, sin duda, uno de tales momentos históricos. Todos los aspectos de la crisis sanitaria y su manejo son materia de debates éticos: la

Cátedra Honorio Delgado, Universidad Peruana Cayetano Heredia. Lima, Perú.

2 Department of Psychiatry, Mayo Clinic School of Medicine. Rochester, MN, EE.UU.

a Médico-Psiquiatra 
actitud, disposición, acciones concretas y decisiones de los médicos y otros profesionales de la salud, las circunstancias del diálogo, la utilización de hospitales, clínicas, salas de emergencia y otras dependencias, la presteza y los textos de medidas administrativas, etc.

Los párrafos siguientes explorarán el tema a punto de partida de los principios rectores de la ética médica, circunscribiéndose luego a aspectos más específicos de la misma, todos sin embargo enfocados primariamente en el análisis y el manejo de aquellos eventos y las implicaciones de la pandemia. Se plantearán las perspectivas diferenciales del enfoque ético en situaciones individuales y en el ámbito de la salud pública para examinar luego las etapas de una implementación sistemática de tales planteamientos en el contexto de la crisis. Al final, se examinará el posible impacto de este enfoque en el manejo de los problemas de salud física y mental de los protagonistas: pacientes y proveedores de atención y servicios médicos.

\section{Principios pertinentes}

Varias organizaciones internacionales de salud han reafirmado el principio básico de la práctica ética: consideración y respeto provistos en igual medida a todo ser humano. Involucrado en un marco de referencia universal, este principio significa que todas las personas cuentan e importan por igual, que sus intereses como seres humanos son o deben ser preocupación prioritaria de los demás y de la sociedad en su conjunto. Desde el punto de vista académico y en regiones como América Latina, estos temas constituyen también indispensables áreas de investigación (2). Sin embargo, en situaciones específicas, la ética del manejo individual postula y acepta un contexto diferente, en función de las características propias de cada paciente. El objetivo esencial es minimizar y corregir el daño que cada persona ha sufrido o sufre y, por ello, es necesario insistir en que todo ser humano involucrado en situaciones problemáticas de salud, debe ser objeto de un cuidado respetuoso y digno $(3,4)$.

Desde un punto de vista general, los principios incluidos en el marco ético de referencia son ocho (57):

1) Respeto: expresado en la provisión de una información consistentemente veraz y sólida, oportunidades uniformes para la expresión de puntos de vista diferentes, consideración apropiada y objetiva de elecciones o decisiones personales.

2) Minimización del daño: orientada a la reducción de su ocurrencia y de la perturbación de actividades esenciales por parte de pacientes y cuidadores, y al aprendizaje de enfoques eficaces.

3) Justeza (fairness): personas con iguales posibilidades de beneficio, resultante de la aplicación de una medida o uso de un recurso de manejo, deben tener igual posibilidad de recibirlo, aun cuando no sería inapropiado preguntar si pueden esperar un tiempo adicional.

4) Trabajo en equipo: apoyo mutuo, asunción y aceptación plena de responsabilidades personales y uso de información apropiadamente compartida.

5) Reciprocidad: profesionales o trabajadores de la salud que acepten mayores demandas o exigencias en su labor, deben recibir adecuado apoyo para ejecutarlas.

6) Sentido de las proporciones: toda información que se comunique debe guardar proporción con los posibles riesgos que su contenido irrogue; asimismo, la eventual restricción de derechos debe ser proporcional a los objetivos de las medidas adoptadas.

7) Flexibilidad: adaptabilidad de los planes de manejo a circunstancias frecuentemente cambiantes.

8) Capacidad y toma de decisiones: deben ser tan abiertas, transparentes, inclusivas y razonables como sea posible. Las decisiones deben ser racionales y prácticas, es decir resultado de un proceso razonable y objetivo, basadas en la evidencia y adaptables a las circunstancias en juego.

Por definición, los principios éticos plantean situaciones de tensión dentro de cada uno y entre todos ellos, por lo que es sabido que, en este campo, no hay respuestas correctas ni equivocadas: el valor de los principios se mide fundamentalmente en función de si su aplicación es éticamente justificable o no.

\section{Acerca de los protagonistas/participantes}

La clásica relación médico-paciente es, una vez más, el eje central del afronte de salud en una situación como la pandemia COVID-19. No puede negarse sin embargo que, al lado de la consideración pluralista (i.e., no individualista o de uno-a-uno) de médicos y otros profesionales o trabajadores de la salud, y de pacientes como recipiendarios de la atención y cuidados de aquéllos, el gran público, la población general se constituye en elemento protagónico sustantivo en casos de emergencia colectiva (8). En otras palabras, el manejo de salud en una pandemia va muchísimo más allá de los límites del domicilio, del grupo familiar o del consultorio para entrar en 
el vasto territorio de la salud pública con medidas y disposiciones radicalmente diferentes porque se aplican a grupos masivos de gente afectada en escenarios que muchas veces no son los vinculados al cuidado médico tradicional (i.e., clínicas de emergencia, hospitales "provisionales", reasignación de unidades clínicas asistenciales e incluso adaptación de escuelas, iglesias, hoteles o parques públicos).

Abundan ejemplos de los cambiante roles de estos protagonistas. De hecho, los estándares de cuidado médico sufren transformaciones radicales cuando la demanda de servicios supera los recursos existentes y fuerza una consideración "más estrictamente utilitaria...(de modo que)...decisiones acerca de cómo responder a necesidades individuales tendrán que ceder el paso a decisiones acerca de cómo maximizar el beneficio global" (7). Los retos de tipo ético que esta realidad plantea son indudablemente enormes.

\section{EI afronte individual}

Queda claro que cada profesional de la salud mantiene su capacidad de decisión cuando enfrenta un caso pero, en situaciones de emergencia, sus acciones profesionales se ven altamente influenciadas por normas éticas que superan la clásica orientación individualista: las llamadas "intuiciones morales cuotidianas" del médico son cuestionadas cuando, por ejemplo, se tiene el caso de un paciente extremadamente enfermo pero situado en medio de la "necesidad cuantitativa" de reducir sustancialmente mortalidad y morbilidad y mantener un vital funcionamiento social. Otro ejemplo es el caso de un paciente "vulnerable" por razones de edad o de padecimiento de una enfermedad crónica vis-a-vis otro con mayores probabilidades de sobrevivencia. Un tercero, el caso de pacientes con compromiso cognitivo y/o capacidad disminuida para la toma de decisiones. La estimación del pronóstico de cada caso individual en base a una variedad de factores se convierte así en sustento claro y determinante de todo tipo de decisiones clínicas $(8,9)$.

La ejecución de tales decisiones debe pues basarse en un examen objetivo de cada caso, un proceso que ni exagere ni minimice la situación y que brinde al paciente y sus allegados la información más precisa posible. Las acciones a tomarse deben, asimismo, guardar debida proporción con el riesgo y los beneficios que puedan producir. Y, como ya se ha indicado varias veces, tales decisiones deben ser consultadas tanto como sea posible, con todas las personas (familiares, amigos, colegas, allegados) vinculadas al caso: la responsabilidad que el profesional de salud asume en tales circunstancias es total por lo que su decisión debe ser reflexiva, no arbitraria, y debe conllevar chances razonables de éxito. A su vez, la documentación médica pertinente debe mantenerse disponible con propósitos claros de explicación, justificación y/o aprendizaje $(4,9-12)$.

\section{Perspectivas colectivas o de Salud Pública}

No es exagerado afirmar que, en situaciones de emergencia sanitaria, las consideraciones colectivas o de salud pública prevalecen sobre las de carácter predominantemente individual. Aun cuando ello no significa en modo alguno que el proceso sea sencillo, hay acuerdo en cuanto a una norma fundamental: si se cuenta con una capacidad radicalmente reducida de recursos para el manejo de todas las serias necesidades de salud en un estado de crisis, es legal y ético que el médico, en seguimiento de apropiadas políticas y protocolos de priorización, que rehúse utilizar un tratamiento potencialmente capaz de salvar la vida de un solo paciente cuando hay otro u otros que califican con prioridad más alta para el mismo tratamiento, por ejemplo, admisión a la Unidad de Cuidados Intensivos: en casos de pacientes con similares posibilidades de sobrevivencia y duración de la hospitalización, debe utilizarse un "enfoque igualitario" que asegure y responda a una asignación justa e imparcial de los recursos (13).

Un problema igualmente crucial es el de la distribución y posibilidades de uso de los recursos, factor decisivo en la privación o suspensión de algunas formas de tratamiento; aparte de que los pacientes continúen recibiendo apoyo emocional y dedicación tan plena como sea posible a su cuidado, tampoco debe faltar un manejo sintomático adecuado; esto debe extenderse, cuando ya sea inevitable, al cuidado del "final de la vida", decisión que afecta muchas veces gravemente tanto a familiares del paciente como a los trabajadores de la salud (médicos, enfermero[a] $\mathrm{s}$, asistente[a]s sociales, practicantes, estudiantes, capellanes, etc.) envueltos en el manejo de cada caso (14). De hecho, éste es un problema de crecientes dimensiones en el manejo global de COVID-19 tal como se traduce en el número de infectados y fallecidos entre trabajadores de salud, cifras debidas tanto a falta de equipos protectores como a las demandas de tiempo dedicado al trabajo diario. El personal de servicio no está obligado a laborar en condiciones frágiles de seguridad y protección $(11,14)$. A su vez, las instituciones hospitalarias deberán reducir o cancelar 
servicios no-esenciales y aumentar el uso de teléfonos, videos, correos electrónicos y medios similares.

Las normas de salud pública en periodos críticos pueden parecer duras e insensibles, situando a aquéllos a cargo de su ejecución y cumplimiento en posiciones sumamente difíciles desde los puntos de vista psicoemocional, social, legal y ético (14-16). Esta situación se hace más evidente debido a la necesaria participación de personal médico adicional (e.g., profesionales ya retirados, estudiantes de medicina de años superiores, voluntarios, etc.) cuyo enorme deseo de ayudar en centros y zonas desposeídas debe ser balanceado por una comprobación clara de su competencia y capacidad, ya que las implicaciones de responsabilidad legal y ética en la toma y ejecución de decisiones son ciertamente significativas $(17,18)$.

\section{Medidas y etapas de implementación}

La gran variedad de desafíos éticos en el curso de una emergencia de salud pública incluye decisiones de diverso orden. Entidades como el Hastings Center (6) plantean una secuencia de deberes destinados al alivio de tensiones entre la práctica clínica tradicional y la de salud pública exigida por situaciones de emergencia. Tal secuencia implica:

El deber de planear, orientado fundamentalmente al manejo de la incertidumbre mediante decisiones en multitud de áreas: nivel de atención (por ej., UCI vs. sala médica general), mantenimiento de funciones vitales esenciales (por ejemplo, uso de ventilador vs. resucitación cardio-pulmonar), suspensión de tratamientos extremos que hayan dado solo resultados negativos o muy pobres, referencias a centros de cuidado paliativo, etc. La cobertura de aspectos logísticos tales como personal disponible, espacios de atención clínica, equipos de protección personal, medicamentos y materiales de uso continuo es igualmente esencial y presenta ángulos éticos innegables (19).

El deber de conferir seguridad o salvaguardar tanto a la fuerza de trabajo (clínicos y personal de apoyo o servicio) laborando en condiciones ocupacionales francamente adversas o riesgosas, como a las llamadas poblaciones vulnerables por razones de edad, historia clínica pasada, pobreza, barreras a la debida atención médica (por ejemplo, carencia de seguro médico, status migratorio, etc.,) (20-22). Personas en entrenamiento convocadas a laborar en estas circunstancias, deben también ser reconocidas como miembros de una población vulnerable.
El deber de guiar que entraña desde asignación de recursos no siempre abundantes hasta un foco definido en la seguridad pública, espectro de acciones que pareciera dejar de lado el interés de casos individuales. Junto a estas situaciones dilemáticas, otras contingencias de la atención médica requieren medidas de prevención ya que es sabido que condiciones de emergencia reducen gradual e inevitablemente la calidad de la atención en diversos escenarios, debido a limitaciones de personal, espacio y equipos $(13,23$, 24).

En el Perú, el Grupo de Ética COVID-19, nombrado por el Ministerio de Salud ha formulado una serie de consideraciones para la toma de decisiones en los servicios de salud durante la pandemia. El documento consta de Principios y Recomendaciones, aquéllos apuntando a objetivos de respeto, determinación, apoyo y justicia, y éstas reafirmando derechos de pacientes y trabajadores de salud, priorizaciones racionales, flexibilidad, comunicación, alivio físico y derecho a una muerte digna si se tornara inevitable (25). La atención a problemas de salud mental y la existencia y función permanentes y cercanas de comités especiales de supervisión en el terreno (para la eventual solución de problemas críticos), y de consultas éticas específicas, deben ser consideraciones explícitas en éste y otros pronunciamientos. El Comité de Ética, dentro del liderazgo de la lucha contra la pandemia, debe asesorar al personal clínico que enfenta incertidumbre y distrés de alto nivel cuando se requieren decisiones vitales en el manejo de las crisis. Una preparación sistemática del personal mediante cursos anuales o semestrales, teóricos y prácticos sobre temas de ética de salud pública y respuesta a desastres, debe ser componente integral y permanente de estas acciones.

\section{DISCUSIÓN}

Los efectos positivos y negativos de la pandemia, agrupados bajo el rubro de impacto socio-cultural (26), entrañan procesos que, de uno u otro modo, han cuestionado estados preexistentes y anuncian cambios más o menos radicales en la conducta colectiva, es decir en el patrimonio cultural de la sociedad global. A su vez, estos cambios conferirán nuevos sesgos y estructuras a la salud mental colectiva $y$, de esa interrelación, emergerán nuevas consideraciones y postulados éticos.

Un ejemplo dramático del vínculo que fenómenos socio-culturales y postulados éticos comparten para 
luego afectar la salud mental, proviene de Argentina, donde se han denunciado "inequidades, infamias y claudicaciones" en aspectos tales como la carencia de equipos de protección, la reducción arbitraria de cobertura por parte de compañías de seguros, la elevación de precios de medicamentos en farmacias y la negligencia casi total de atención a gente pobre, desvalida, marginada y, por todo ello, sustancialmente vulnerable (27).

Las llamadas políticas sociales punitivas, fenómeno in crescendo en países como Estados Unidos, Brasil, Argentina, España e Italia nutren resentimientos xenofóbicos y violencia concomitante directa o indirectamente influenciada por gobiernos autoritarios (28-31). Otra expresión de esta actitud emocional es la ocurrencia de inequidades de declarada base racial tan sutil como "diferencias biológicas en los órganos respiratorios de gentes de raza negra y blanca" $(32,33)$, o tan cruda como la descripción de "conductas estereotipadas" y de "estigmatización territorial" en grupos minoritarios (21).

Las implicaciones éticas de "sub-servicios" o de atención deficiente de salud mental a los segmentos más vulnerables de la población, ya de por sí deplorables en periodos de relativa calma, se acentúan en crisis como la pandemia viral. Se habla de "leyes invertidas de atención médica" (16), el casi total abandono del cuidado de pacientes con enfermedades mentales severas debido a las exigencias de atención de las víctimas primarias de COVID-19 --no justificable ni siquiera en base a la preferencia del cuidado de aquéllos con "mejor pronóstico" ya que estamos hablando de condiciones de naturaleza totalmente distinta; esta situación se agrava cuando son los propios pacientes mentales los que muestran síntomas de la infección viral. Otros grupos vulnerables incluyen pacientes con problemas de aprendizaje y trastornos del neurodesarrollo, al igual que grupos de "exclusión social" como reclusos, refugiados o los "sin techo" (34-36).

\section{CONCLUSIONES}

La pandemia de COVID-19 ha echado las bases de una nueva cultura social y ha planteado desafíos éticos trascendentales. A menos que la humanidad utilice masivos volúmenes de negación, ignorancia u olvido intencional, una nueva definición de normalidad adquirirá forma en los años y lustros por venir $(37,38)$. Cada aspecto de esta nueva configuración existencial tendrá repercusiones en el campo de la salud mental individual y colectiva, configurando un escenario aún más complicado porque cambios de tipo socio-cultural y principios éticos (en medicina y en cualquier otro campo) poseen agendas potencialmente conflictivas como se ha visto en diversos pasajes de este trabajo.

Lo anterior significa que, en situaciones futuras (ya avizoradas en la pandemia de COVID-19), la perspectiva ética, particularmente la que tiene que ver con salud pública, puede colisionar seriamente con las nuevas realidades socio-culturales y devenir entonces en problemas emocionales mucho más marcados $(2$, 39-41). En otras palabras y a despecho de toda otra consideración, no es ni será posible hablar de una aplicabilidad general de normas éticas ya que, en última instancia, cada caso, cada situación individual puede $y / 0$ debe ser materia de enfoques singulares. Se trata, en suma, de problemas ambiguos que, por lo mismo, pueden dar lugar a soluciones insuficientes. El futuro, urgentemente convocado por la crisis pandémica, solo puede esperar preguntas mejor planteadas y arduo trabajo de estudiosos, autoridades, individuos y sociedad, en procura de respuestas más consistentes.

\section{Correspondencia}

Renato D. Alarcón-Guzmán

1 Lakeside Dr. Apt. 1602, Oakland, CA 94612, USA

Correo electrónico: renato.alarcon@upch.pe / Alarcon. Renato@mayo.edu

\section{Declaraciones}

El autor declara no haber recibido financiamiento alguno para la preparación del artículo y no tener conflicto de interés económico, institucional, laboral o personal.

\section{REFERENCIAS BIBLIOGRÁFICAS}

1. Drane JF (Editor). Bioethical Perspectives from Ibero-America. J Med Philosophy. 1996; 21(6):557569. DOI: https://doi.org/10.1093/jmp/21.6.557

2. Pessini L, de Barchifontaine $\mathrm{C}$ de $\mathrm{P}$, Lolas F. (Coordinadores). Perspectivas de la Bioética en Iberoamérica (Programa de Bioética de la Organización Panamericana de la Salud). Santiago de Chile: Andros Impresores; 2007.

3. World Psychiatric Association. Special update from the President: COVID-19. Geneva: World Psychiatric Association; Updated, March 20, 2020.

4. Italian Society of Psychiatry. Recommendations for Mental Health Departments regarding activities and measures of contrast and containment of the SARS- 
COV-19 virus. Evidence Based Psychiatric Care. March 20,2020. Disponible en: https://www.evidencebased-psychiatric-care.org/wp-content/ uploads/2020/04/SARS-COV-19_Suppl_Special_ Rivista_SIP_eng.pdf 28/05/2020

5. Department of Health. Responding to pandemic influenza - The ethical framework for policy and planning. London: Department of Health; November 2007.

6. Berlinger N, Wynia M, Powell T, Hester M, Milliken A, Fabi R, et al. Ethical framework for Health Care institutions responding to novel Coronavirus SARS-CoV-2 (COVID-19). Guidelines for institutional Ethics services responding to COVID-19. New York: The Hastings Center; March 16, 2020. Disponible en: thehastingscenter. org/ethicalframeworkcovid19

7. British General Medical Council. COVID-19. Ethical Issues: A guidance note. London, UK: British General Medical Council; March 3, 2020.

8. Cipiriani A, Ostinelli E, Smith K, Macdonald O, Sheriff, Harrison P, et al. COVID-19 and its impact on mental health patients, staff and services: systematic review with recommendations for clinical practice and research. Prospero. 2020. Disponible en: https://www.crd.york.ac.uk/prospero/display_ record.php?ID=CRD42020178819 28/05/2020

9. The Lancet. Redefining vulnerability in the era of COVID-19. Lancet. 2020; 395(10230): 1089. DOI: https://doi.org/10.1016/S0140-6736(20)30757-1

10. Chowell G, Mizumoto K. The COVID-19 pandemic in the USA: what might we expect?. Comment, The Lancet. 2020; 395: 0-0.

11. García PJ. ¿Qué sucederá ahora? Lima, Perú: Diario El Comercio; Marzo 21, 2020.

12. Kirkwood T. Health, wellness and their determinants. Hektoen International. 2020. Disponible en: https:// hekint.org/2020/01/28/health-wellness-and-theirdeterminants/ 28/05/2020

13. Emanuel EJ, Persad G, Upshur R, Thome B, Parker M, Glickman A, et al. Fair allocation of scarce medical resources in the time of Covid-19. N Engl $\mathrm{J}$ Med 2020; 382:2049-2055. DOI: 10.1056/NEJMsb20 05114

14. Rose S. Medical Student education in the time of COVID-19. JAMA. March 31, 2020. doi: 10.1001/ jama.2020.5227

15. Fauci AS, Lane HC, Redfield RR. Covid-19 Navigating the uncharted. NEJM, 2020; 382: 12681269.

16. The Lancet Psychiatry. Mental health and COVID-19: change the conversation. Lancet Psychiatry. 2020 Jun;7(6):463. doi: 10.1016/S2215-0366(20)30194-2

17. Toner E, Waldhorn R. What US hospitals should do now to prepare for a COVID-19 pandemic. Baltimore, MD: Johns Hopkins University Center for Health
Security; 2020. Disponible en: https://www.centerfor healthsecurity.org/cbn/2020/cbnreport-02272020 28/05/2020

18. Hick JL, Hanfling D, Wynia MK, Pavia AT. Duty to plan: health care, crisis standards of care, and novel coronavirus SARS-CoV-2. NAM Perspectives. March 5, 2020. Disponible en: https://nam.edu/ duty-to-plan-health-care-crisis-standards-of-careand-novel-coronavirus-sars-cov-2/

19. Vigo D, Patten S, Pajer K, Krausz M, Taylor S, Rush B, et al. Mental Health of communities during the COVID-19 Pandemic. Canad J Psychiatry. 2020. DOI: https://doi.org/10.1177/0706743720926676

20. The Lancet. COVID-19 will not leave behind refugee and migrants. Lancet. April 4, 2020; 395: 1090.

21. Chowkwanyun M, Reed AL. Racial health disparities and Covid-19 - Caution and context. NEJM, May 6, 2020. DOI: 10.1056/NEJMp2012910

22. Van Dorn A, Cooney RE, Sabin ML. COVID-19 exacerbating inequalities in the US. The Lancet. 2020; 395: 1243-244.

23. Fineberg HV. Ten Weeks to crush the curve. N Engl J Med. 2020;382(17):e37. doi: 10.1056/NEJMe20072 63

24. Shonkoff JP, Williams DR. Thinking about racial disparities in COVID-19 impacts through a scienceinformed, early childhood lens. Center for the developing child at Harvard University. Published April 27, 2020. Disponible en: https:// developingchild.harvard.edu/thinking-about-racialdisparities-in-covid-19-impacts-through-a-scienceinformed-early-childhood-lens/ 28/05/2020

25. Ministerio de Salud; Grupo de Ética Covid-19. Consideraciones éticas para la toma de decisiones en los Servicios de Salud durante la pandemia COVID-19. Lima: Ministerio de Salud; Abril 30, 2020.

26. Alarcón RD. Impacto socio-cultural y mental de la pandemia COVID-19. Rev Per Med Exper Salud Pública, 2020. (En revisión).

27. Levin S. Pandemia por coronavirus: inequidades, infamias y claudicaciones. Buenos Aires: Asociación de Psiquiatras Argentinos; Mayo 3, 2020.

28. Pericás JM. Authoritarianism and the threat of infectious disease. Lancet. 2020; 395: 1111-1112.

29. Laughland O. "A perfect storm": poverty and race add to Covid-19 toll in US deep south. London, UK: The Guardian; April 12, 2020. Disponible en: 28/05/2020 https://www.theguardian.com/us-news/ 2020/apr/12/coronavirus-us-deep-south-povertyrace-perfect-storm 28/05/2020

30. Meyer EPN, Bustamante T. Authoritarianism without emergency powers: Brazil under COVID-19. VerfBlog. April 8, 2020. Disponible en: https:// verfassungblog.de/authoritarianism-withoutemergency-powers-brazil-under-covid-19/ 
$28 / 05 / 2020$

31. Bustamante T, Meyer EPN. Bolsonarism and Covid-19: Truth strikes back. Int J Const L Blog, March 24, 2020. Disponible en: http://www. iconnectblog.com/2020/03/bolsonarism-and-covid19-truth-strikes-back/ 28/05/2020

32. Braun L. Breathing race into the machine: the surprising career of the spirometer from plantation to genetics. Minneapolis, MN: University of Minnesota Press; 2014.

33. White AIR. Historical linkages: epidemic threat, economic risk, and xenophobia. Lancet. April 18, 2020; 395: 1250-251.

34. Ornell F, Schuch JB, Sordi AO, Paim Kessler FH. "Pandemic fear" and COVID-19: mental health burden and strategies. Braz J Psychiatry. 2020;42(3):232-235. doi: 10.1590/1516-4446-20200008

35. Tsai J, Wilson M. COVID-19: a potential public health problem for homeless populations. Lancet Public Health. 2020; S2468-2667(20): 30053-300560 DOI: $10.1016 / \mathrm{S} 2468-2667(20) 30053-0$

36. Hughes JF. How COVID-19 closures will irrevocably change our lives. Physician Sense, April 24, 2020.
Disponible en: https://www.mdlinx.com/ physiciansense/how-covid-19-closures-willirrevocably-change-our-lives/ 28/05/2020

37. Smith K, Ostinelli E, Cipriani A. Covid-19 and mental health: A transformational opportunity to apply an evidence-based approach to clinical practice and research. Evid Based Ment Health. 2020;23(2):4546. DOI: 10.1136/ebmental-2020-300155

38. Zilber C. Surreal boundary shifts during a pandemic. Psychiatric News. Washington DC. Published online Abril 9, 2020. DOI: https://doi.org/10.1176/appi. pn.2020.4b25

39. World Association of Social Psychiatry. WASP Position Statement on the Coronavirus Disease (COVID-19) Pandemic. London: World Association of Social Psychiatry; Abril 2020.

40. Edwards RB (Editor). Psychiatry and Ethics. Insanity, rational autonomy, and mental health care. Buffalo, NY: Prometheus Books; 1982.

41. Pfefferbaum B, North CS. Mental Health and the Covid-19 Pandemic. New Eng J Med. Abril 13, 2020. DOI: 10.1056/NEJMp2008017 